\title{
Sudden unexpected death in an infant with L-2-hydroxyglutaric aciduria
}

\author{
Marine Jequier Gygax • Eliane Roulet-Perez • \\ Kathleen Meagher-Villemure • Cornelis Jakobs • \\ Gajja S. Salomons • Olivier Boulat • \\ Andrea Superti-Furga $\cdot$ Diana Ballhausen • \\ Luisa Bonafé
}

Received: 9 July 2008 /Revised: 16 October 2008 /Accepted: 22 October 2008 /Published online: 13 November 2008

(C) Springer-Verlag 2008

\begin{abstract}
Inherited metabolic disorders are the cause of a small but significant number of sudden unexpected deaths in infancy. We report a girl who suddenly died at 11 months of age, during an intercurrent illness. Autopsy showed spongiform lesions in the subcortical white matter, in the basal ganglia, and in the dentate nuclei. Investigations in an older sister with developmental delay, ataxia, and tremor revealed L-2-hydroxyglutaric aciduria and subcortical white matter changes with hyperintensity of the basal ganglia and
\end{abstract}

M. Jequier Gygax $\cdot$ E. Roulet-Perez

Child Neurology Unit, Department of Pediatrics,

Centre Hospitalier Universitaire Vaudois,

Lausanne, Switzerland

K. Meagher-Villemure

Institute of Pathology, Centre Hospitalier Universitaire Vaudois,

Lausanne, Switzerland

C. Jakobs $\cdot$ G. S. Salomons

Metabolic Unit, Department of Clinical Chemistry,

VU University Medical Center,

Amsterdam, The Netherlands

O. Boulat

Clinical Chemistry Laboratory,

Centre Hospitalier Universitaire Vaudois,

Lausanne, Switzerland

A. Superti-Furga $\cdot$ D. Ballhausen $\cdot$ L. Bonafé $(\bowtie)$

Division of Molecular Pediatrics,

Centre Hospitalier Universitaire Vaudois,

Clinique Infantile 02-35 Av. P. Decker 2,

1011 Lausanne, Switzerland

e-mail: Luisa.Bonafe@chuv.ch

\section{A. Superti-Furga}

Department of Pediatrics and Adolescent Medicine,

University of Freiburg,

Freiburg, Germany dentate nuclei at brain magnetic resonance imaging. Both children were homozygous for a splice site mutation in the L2HGDH gene. Sudden death has not been reported in association with L-2-hydroxyglutaric aciduria so far, but since this inborn error of metabolism is potentially treatable, early diagnosis may be important.

Keywords L-2-Hydroxyglutarate · Sudden death · SIDS . SUDI · Organic aciduria

\section{Introduction}

L-2-Hydroxyglutaric aciduria (OMIM 236792) is an inborn error of metabolism characterized by the accumulation of L-2-hydroxyglutaric acid in body fluids [3, 13]. Since the first description of L-2-hydroxyglutaric aciduria in 1980 [13], less than 100 cases have been reported $[2,4,5,6,8$ $11,14-16,18,20,21,23,26,27,29,38,41-45,47,48]$. Common clinical features include developmental delay, macrocephaly, ataxia, pyramidal signs, and seizures. The clinical course is usually slowly progressive, without acute phases of neurological deterioration. Brain magnetic resonance imaging (MRI) shows bilateral involvement of the pallidum, the dentate nuclei, and subcortical white matter [1, 8, 28, 35-37]. Neuropathology descriptions mainly report spongiosis of the subcortical white matter with cystic cavitations [20,35], diffuse astrocytosis predominating in the cerebral and cerebellar white matter [5], gliosis, and neuronal loss in the dentate nuclei $[5,35]$. Interestingly, brain tumors seem to occur with increased frequency in L-2-hydroxyglutaric aciduria and represent a possible cause of death $[2,19,24,45]$; the mechanism of this unusual phenomenon is unclear. A very severe form of L-2- 
hydroxyglutaric aciduria with neonatal death has been reported by Chen and coauthors [5]. Otherwise, no sudden infant death has been reported in L-2-hydroxyglutaric aciduria so far, and this disorder is not listed among the several inherited metabolic disorders associated with sudden unexpected death in infancy (SUDI) [7, 25]. This paper describes two siblings with L-2-hydroxyglutaric aciduria, presenting with two drastically different clinical courses, and reports the first instance of association of this inborn error of metabolism with SUDI.

\section{Case reports}

Patient 1 was an 11-month-old girl, born to consanguineous parents of Tamil origin. She was the third child after an 11-year-old healthy sister and a 9-year-old sister (patient 2). The father suffers from type 1 diabetes; the family history is otherwise unremarkable.

Pregnancy and delivery were uneventful. Growth and psychomotor development were fully normal until age 10 months, when she presented, within $10 \mathrm{~h}$, three episodes of activity arrest and staring during a few minutes. Each episode was followed by post-ictal sleep and full recovery. She did not present any other sign of illness and had no fever. Clinical examination, including neurological status and fundoscopy, was perfectly normal between the episodes. Her weight was $10.6 \mathrm{~kg}$ (P90), length $75 \mathrm{~cm}$ (P50), and fronto-occipital head circumference $47 \mathrm{~cm}$ (P90-97). Routine blood, urine, and cerebrospinal fluid (CSF) investigations, as well as cardiac investigations and microbiological screening were negative. Electroencephalography (EEG) showed non-specific posterior slow waves without epileptiform discharges. One week later, during an intercurrent febrile illness with diarrhea since $48 \mathrm{~h}$, she suddenly died while sleeping in her mother's arm. The mother reported no abnormal eye or limb movement and no respiratory distress. She was examined a few hours earlier at the pediatric hospital, and the clinical examination did not show any sign of severe infection; there was no dehydration and no neurological abnormality.

Pathology A complete autopsy was performed in accordance to national regulations for unexpected death. Samples from every organ were collected after dissection, fixed in formalin, and stained with hematoxylin-eosin. Complete body X-ray, samples from blood, vitreous, muscle, and gastric content were submitted for toxicologic tests as well as bacteriology and virology cultures from blood, lungs, and liver tissues; all these tests resulted to be normal. The autopsy revealed that all organs were normal in size and structure. There was no evidence of trauma, malformation, malignancy, or infection. Brain weight was $1,032 \mathrm{~g}$, slightly higher than expected for age (normal range, 900-950 g). On visual inspection of the central nervous system, the cerebral conformation was normal, and no herniation was seen. On coronal sections, the ventricular system was not enlarged, and there was normal appearance of the gray and white matter. On histology, there was bilateral striking vacuolation in the subcortical white matter (Figs. 1a,b), around and within the putamen, in the dentate nuclei and surrounding in the cerebellum (Fig. 1c). The cerebellar folia were unremarkable. In all involved areas, the spongiosis was accompanied by significant astrogliosis. The vacuoles were mainly seen in the myelin sheaths (Fig. 1d). There was no evidence of neuronal loss, no axonal damage, and no significant vascular changes.

Molecular investigations Genomic DNA was extracted from a frozen muscle specimen, collected during autopsy. Molecular analysis in the $L 2 H G D H$ gene was performed after the diagnosis was made in patient 2 , and revealed the homozygous splice site mutation c. $738+1 \mathrm{G}>\mathrm{A}(\mathrm{IVS} 6+1 \mathrm{G}>\mathrm{A})$; both parents were carriers of this mutation.

Patient 2 is the 9-year-old sister of patient 1 . She was born at term after an uneventful pregnancy and delivery. Psychomotor development was slightly delayed in the first years of life, with walking at 18 months and language delay. At age 3 and 4 years, she presented two episodes of uncomplicated generalized febrile convulsions. She was seen for the first time at the age of 6 years: She presented with nonspecific global developmental delay with motor clumsiness, without pyramidal signs. From age 6 to 9 years, she progressively developed action tremor, light gait ataxia, dysarthria and drooling, and moderate mental retardation. No neurological deterioration was reported during febrile illness. Several attempts to obtain brain imaging failed, and further investigations were not possible because of lack of compliance.

One month after the death of her younger sister, during a viral infection, she presented a unique episode of activity arrest with hypotonia and upward eye deviation, followed by post-ictal sleep. Neurological examination showed gait ataxia with clear imbalance at cerebellar testing, action and intention tremor, and dysarthria. Cranial nerves and deep tendon reflexes were normal. Fundoscopy did not show any papillary edema. Her weight was $33 \mathrm{~kg}$ (P75), height $136 \mathrm{~cm}$ (P50-75), and fronto-occipital head circumference $53.8 \mathrm{~cm}$ (P90-97). The clinical examination was otherwise normal. EEG revealed nonspecific excess of slow waves at the vertex without epileptiform discharges.

Neuroradiological investigation Brain MRI at the age of 9 years showed predominantly frontal subcortical white matter hyperintensity on T2 sequences, with abnormal 
Fig. 1 Brain histopathology of patient 1: a section of cerebral cortex and white matter showing subcortical spongiosis (arrow) and vascular congestion. No significant myelin loss. HE-LFB $20 \times$. Insert Immunohistochemistry with glial fibrillary acidic protein (GFAP) revealing strong positive reaction of astrogliosis at the subcortical level. GFAP 20×. b Higher power level showing the numerous vacuoles (arrow) in the subcortical matter. HE 200×. c Section of cerebellum with marked spongiosis (arrow) within the dentate nucleus, no abnormality within the cerebellar folia. HE-LFB 40×. d High power of cerebellar white matter near the dentate nucleus showing marked vacuolation (arrow) within the myelin sheaths. HE-LFB 400×. Insert Immunohistochemistry with GFAP revealing the significant astrogliosis surrounding the vacuolated white matter
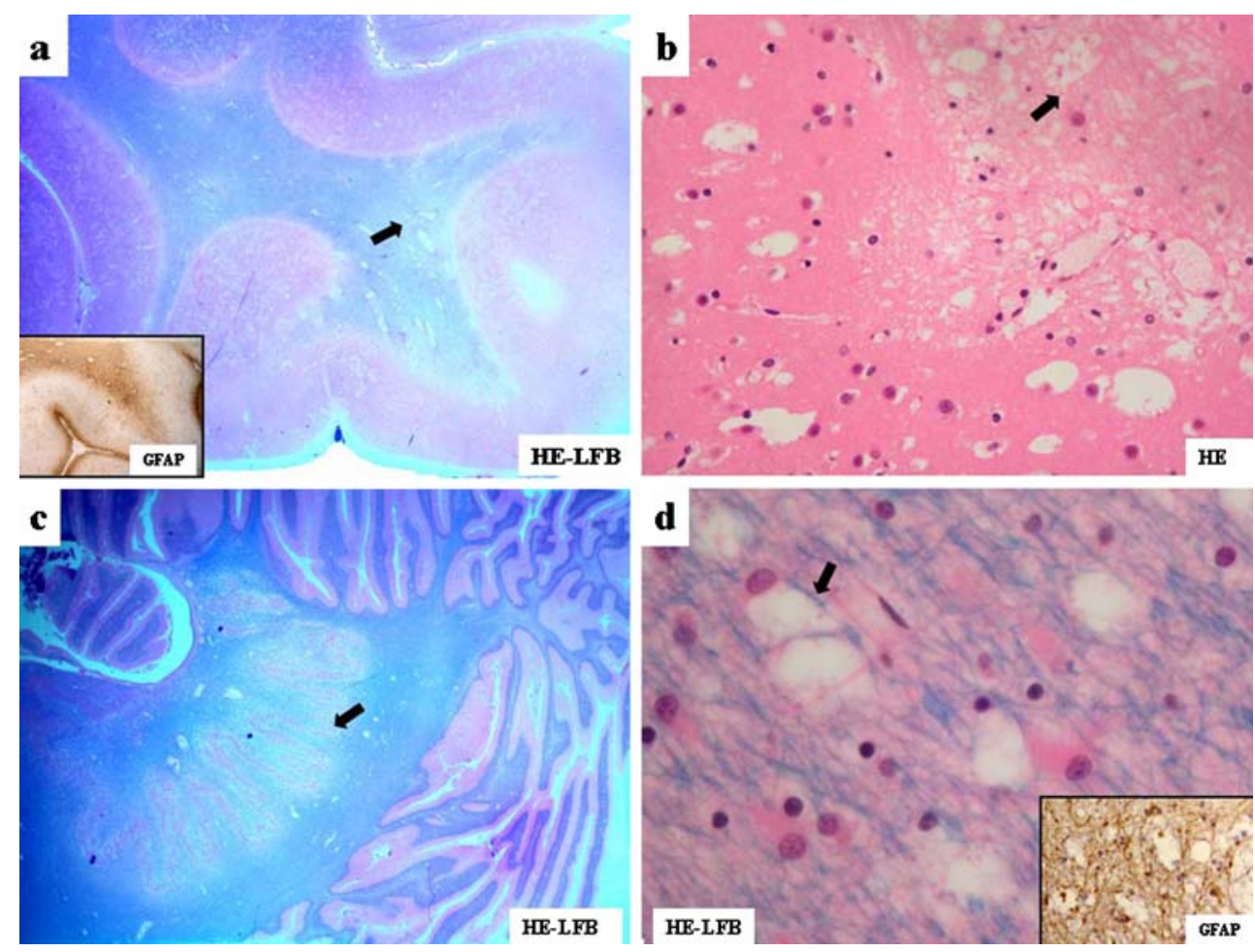

signal in bilateral dentate nuclei and slight signal alteration in the basal ganglia (Fig. 2). Standard multivoxel and monovoxel proton spectroscopy was normal.

Biochemical and molecular investigations Routine blood, urine, and CSF analysis were normal. Acylcarnitines profile was normal, as well as carbohydrate-deficient glycoprotein analysis. Urinary organic acids analysis in multiple urine samples revealed markedly increased excretion of 2-hydroxyglutarate (1612-2477 $\mathrm{mmol} / \mathrm{mol}$ creatinine; normal range $<15.4)$. Enantiomeric separation [40] confirmed that most of the excreted 2-hydroxyglutarate was in its L-form (L-2-hydroxyglutarate 1,580 $\mathrm{mmol} / \mathrm{mol}$ creatinine), whereas D-2-hydroxyglutarate excretion was within the control range. Plasma amino acids showed increased lysine levels (359 $\mu \mathrm{mol} / 1$; normal range, 116-250). CSF analysis showed markedly increased 2hydroxyglutarate $(51 \mu \mathrm{mol} / \mathrm{l}$; normal range, $<3)$ and lysine (72 $\mu \mathrm{mol} / \mathrm{l}$; normal range, 15-37). Genomic DNA was extracted from peripheral blood and molecular analysis in the $L 2 H G D H$ gene revealed the same homozygous splice site mutation found in patient 1 (IVS6 $+1 \mathrm{G}>\mathrm{A}$ ).

Treatment Therapeutic trials were performed with oral coenzyme Q10 (400 mg/day) during 6 months and, more recently, with oral riboflavin (200 $\mathrm{mg}$ /day) during 2 months. L-2Hydroxyglutarate in urine and lysine in plasma were monitored every 4 weeks during both trials, and no significant reduction was observed. CSF L-2-hydroxyglutarate was measured in CSF before and during treatment, without any significant change. Brain MRI findings remained unchanged before and after the trial with coenzyme Q10. Clinical neurological assessment was unchanged before, during, and after the trials with both substances.

\section{Discussion}

While the clinical presentation of patient 2 with early-onset delay of psychomotor milestones, ataxia, tremor, and slowly progressive clinical course is similar to the majority of the reported cases, her sibling had normal psychomotor development until sudden unexpected death at 11 months, during a mild intercurrent illness. Neuropathology findings of patient 1 corresponded well to the neuroradiological changes of patient 2 , despite very different clinical courses. Current knowledge on the molecular bases of L-2-hydroxyglutaric aciduria shows no clear correlation between genotype and phenotype [34] and no correlation between neuroradiological images, severity of the disease, and prognosis. The family presented in this paper, the first reported of Tamil origin, segregates a previously undescribed mutation in the universally conserved GT dinucleotide at a donor splice site in the L2HGDH gene that is predicted to affect splicing like in two other reported patients [43]. Our report confirms intrafamilial phenotypic variability despite identical genotypes and lack of correlation between the extent of brain lesions and clinical signs and symptoms.

Clinical heterogeneity in affected siblings is known in L-2-hydroxyglutaric aciduria [9, 15], but sudden death has 
Fig. 2 Brain MRI of patient 2 at age 9 years: a hyperintensity of the subcortical white matter (upper arrows) and dentate nuclei (lower arrow) in T2 ponderation; b slight signal alteration of the basal ganglia (arrow) in $\mathrm{T} 2$ ponderation
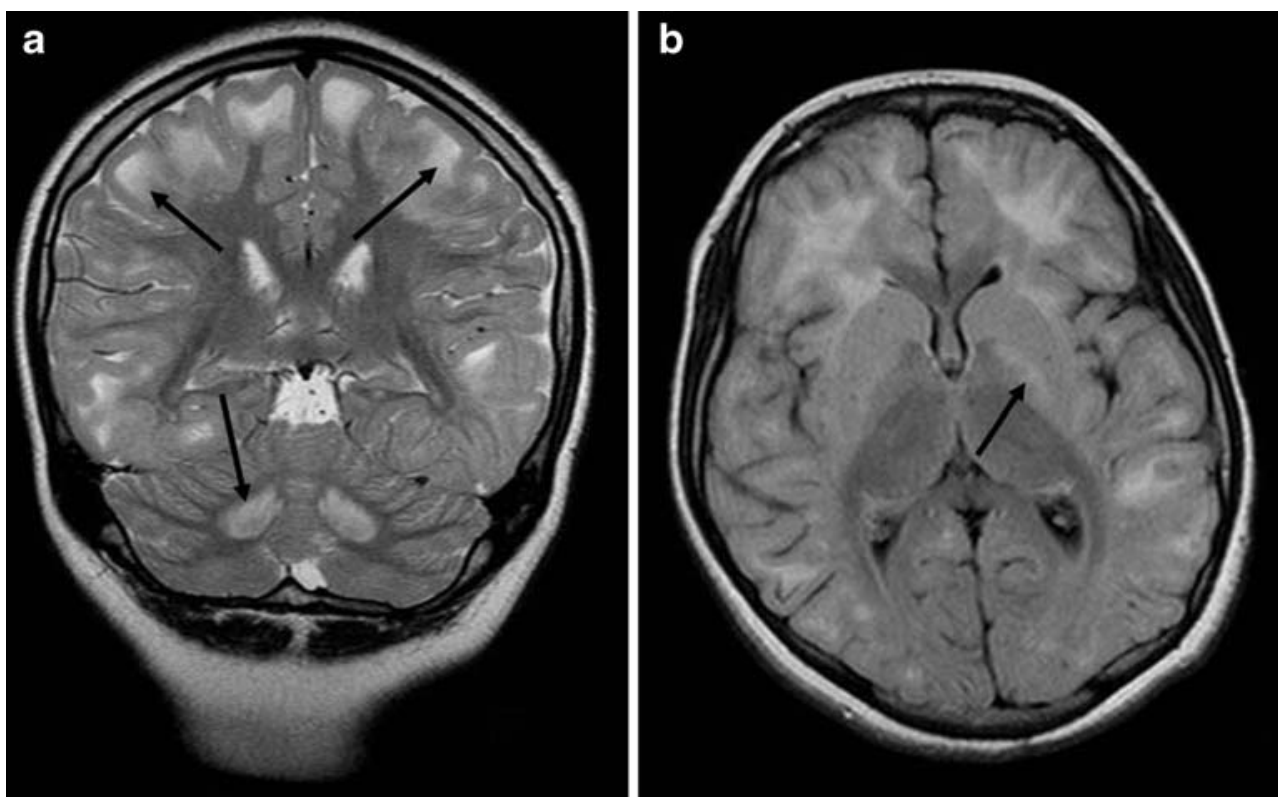

not been previously described in this disorder. This unexpected observation raises questions concerning the primary pathogenetic process. L-2-Hydroxyglutaric aciduria is caused by deficient activity of the flavin adenine dinucleotide (FAD)-dependent enzyme L-2-hydroxyglutaric dehydrogenase, which converts L-2-hydroxyglutarate to alpha-ketoglutarate $[30,31]$. It has been proposed that L-2-hydroxyglutaric aciduria is a "metabolite repair" disorder [32], in which L-2-hydroxyglutarate, a byproduct of the Krebs cycle, fails to be re-converted in its own precursor (alpha-ketoglutarate). Both the toxic effect of accumulated L-2-hydroxyglutarate [39] and alpha-ketoglutarate depletion [17] are currently considered as possible pathogenetic mechanisms. Reduced availability of alpha-ketoglutarate for the Krebs cycle may cause a major derangement in energy metabolism, which seems to be the common pathogenetic mechanism of organic acidurias known to be associated to SUDI, although the final molecular mechanism triggering sudden death remains unknown.

To date, only one case of infant death associated with L-2-hydroxyglutaric aciduria has been reported [5]; however, the patient was critically ill, and the early death was not unexpected, as there was severe neonatal encephalopathy and rapid deterioration at 1 month of age. Three other cases of death in childhood were attributed to development of cerebral tumors [2, 24, 45]. This is the first report of unexpected death without pre-existing clinical signs in a child with L-2-hydroxyglutaric aciduria. Autopsy findings give no argument for overwhelming infectious disease, although it cannot formally be excluded. Pathology studies exclude the presence of brain tumor and the involvement of any other organ as the ultimate cause of death. Brain histopathological abnormalities are similar to the previously reported cases [5, 20, 35] and do not allow to point at a specific brain lesion as the cause of death. In contrast to the other reported cases of infancy/childhood death in L-2hydroxyglutaric aciduria, our patient's clinical presentation is that of a SUDI, in terms of age, death occurring during sleep, and concomitant associated diagnosis of a metabolic disorder [7]. However, until further cases are reported, it cannot be determined whether this association is causative or coincidental. Inborn errors of metabolism have been diagnosed in $2 \%$ to $5 \%$ of SUDI [12] but may remain underdiagnosed in centres lacking expertise in pediatric pathology and metabolic investigation protocols [22]. The importance of a protocol for the laboratory investigation of SUDI has been highlighted by Olpin and coauthors [25]; however, post-mortem diagnosis of an inborn error of metabolism is often difficult due to the sensitivity of biochemical analysis in body fluids or tissues to proteolytic deterioration of inner organs. When urine and/or CSF are available, organic acid analysis by gas chromatography mass spectrometry is the best test to diagnose L-2hydroxyglutaric aciduria. Although rare, the diagnosis of a metabolic disorder in SUDI allows not only appropriate genetic and reproductive counseling but also the detection of affected siblings and, for some disorders, appropriate treatment.

Until recently, there was no effective specific therapy for L-2-hydroxyglutaric aciduria. Two very recent reports indicate the possible therapeutic role of FAD [33], cofactor of the enzyme, and of its precursor riboflavin [46]. We could not confirm this observation in patient 2 . However, if the spectacular clinical improvement of L-2-hydroxyglutaric aciduria patients under riboflavin/FAD treatment observed in these first reports will be confirmed in further cases, 
early identification of this inborn error of metabolism in affected families may result in a better neurological prognosis and possibly in prevention of SUDI.

Acknowledgments We thank Dr P. Maeder for the interpretation of brain MRI.

\section{References}

1. Aydin K, Ozmen M, Tatli B et al (2003) Single-voxel MR spectroscopy and diffusion-weighted MRI in two patients with 1-2-hydroxyglutaric aciduria. Pediatr Radiol 33:872-876. doi:10.1007/s00247-003-1029-Z

2. Barbot C, Fineza I, Diogo L et al (1997) L-2-Hydroxyglutaric aciduria: clinical, biochemical and magnetic resonance imaging in six Portuguese pediatric patients. Brain Dev 19:268-273. doi:10.1016/S0387-7604(97)00574-3

3. Barth PG, Hoffmann GF, Jaeken J et al (1992) L-2-hydroxyglutaric acidemia: a novel inherited neurometabolic disease. Ann Neurol 32:66-71. doi:10.1002/ana.410320111

4. Barth PG, Hoffmann GF, Jaeken J et al (1993) L-2-hydroxyglutaric acidaemia: clinical and biochemical findings in 12 patients and preliminary report on L-2-hydroxyacid dehydrogenase. J Inherit Metab Dis 16:753-761. doi:10.1007/BF00711907

5. Chen E, Nyhan WL, Jakobs C et al (1996) L-2-Hydroxyglutaric aciduria: neuropathological correlations and first report of severe neurodegenerative disease and neonatal death. J Inherit Metab Dis 19:335-343. doi:10.1007/BF01799264

6. Clerc C, Bataillard M, Richard P et al (2000) An adult form of L-2-hydroxyglutaric aciduria revealed by tremor. Eur Neurol 43:119-120. doi: $10.1159 / 000008149$

7. Cote A, Russo P, Michaud J (1999) Sudden unexpected deaths in infancy: what are the causes? J Pediatr 135:437-443. doi:10.1016/ S0022-3476(99)70165-4

8. D'Incerti L, Farina L, Moroni I et al (1998) L-2-Hydroxyglutaric aciduria: MRI in seven cases. Neuroradiology 40:727-733. doi:10.1007/s002340050673

9. de Klerk JB, Huijmans JG, Stroink H et al (1997) L-2hydroxyglutaric aciduria: clinical heterogeneity versus biochemical homogeneity in a sibship. Neuropediatrics 28:314-317. doi:10.1055/ s-2007-973722

10. Diogo L, Fineza I, Canha J et al (1996) Macrocephaly as the presenting feature of L-2-hydroxyglutaric aciduria in a 5-month-old boy. J Inherit Metab Dis 19:369-370. doi:10.1007/BF01799270

11. Divry P, Jakobs C, Vianey-Saban C et al (1993) L-2-hydroxyglutaric aciduria: two further cases. J Inherit Metab Dis 16:505-507. doi:10.1007/BF00711666

12. Dott M, Chace D, Fierro M et al (2006) Metabolic disorders detectable by tandem mass spectrometry and unexpected early childhood mortality: a population-based study. Am J Med Genet A 140:837-842 . doi:10.1002/ajmg.a.31180

13. Duran M, Kamerling JP, Bakker HD et al (1980) L-2-Hydroxyglutaric aciduria: an inborn error of metabolism? J Inherit Metab Dis 3:109-112. doi:10.1007/BF02312543

14. Fujitake J, Ishikawa Y, Fujii H et al (1999) L-2-hydroxyglutaric aciduria: two Japanese adult cases in one family. J Neurol 246:378-382. doi:10.1007/s004150050367

15. Goffette SM, Duprez TP, Nassogne MC et al (2006) L-2Hydroxyglutaric aciduria: clinical, genetic, and brain MRI characteristics in two adult sisters. Eur J Neurol 13:499-504. doi:10.1111/j.1468-1331.2006.01282.x
16. Kaabachi N, Larnaout A, Rabier D et al (1993) Familial encephalopathy and L-2-hydroxyglutaric aciduria. J Inherit Metab Dis 16:893. doi:10.1007/BF00714285

17. Kamoun P, Richard V, Rabier D et al (2002) Plasma lysine concentration and availability of 2-ketoglutarate in liver mitochondria. J Inherit Metab Dis 25:1-6. doi:10.1023/A:1015195009330

18. Kossoff EH, Keswani SC, Raymond GV (2001) L-2-hydroxyglutaric aciduria presenting as migraine. Neurology 57:1731-1732

19. Larnaout A, Amouri R, Neji S et al (2007) Osteoma of the calvaria in L-2-hydroxyglutaric aciduria. J Inherit Metab Dis 30:980. doi:10.1007/s10545-007-0576-0

20. Larnaout A, Hentati F, Belal S et al (1994) Clinical and pathological study of three Tunisian siblings with L-2-hydroxyglutaric aciduria. Acta Neuropathol 88:367-370. doi:10.1007/BF00310381

21. Lee C, Born M, Salomons GS et al (2006) Hemiconvulsionhemiplegia-epilepsy syndrome as a presenting feature of L-2hydroxyglutaric aciduria. J Child Neurol 21:538-540

22. Loughrey CM, Preece MA, Green A (2005) Sudden unexpected death in infancy (SUDI). J Clin Pathol 58:20-21. doi:10.1136/ jcp.2004.020677

23. Mahfoud A, Dominguez CL, Perez A et al (2004) L-2hydroxyglutaric aciduria: clinical, biochemical and neuroradiological findings in two Venezuelan patients. Rev Neurol 39:343-346

24. Moroni I, Bugiani M, D’Incerti L et al (2004) L-2-hydroxyglutaric aciduria and brain malignant tumors: a predisposing condition? Neurology 62:1882-1884

25. Olpin SE (2004) The metabolic investigation of sudden infant death. Ann Clin Biochem 41:282-293. doi:10.1258/0004563041201473

26. Owens WE, Okun MS (2004) Dystonia, tremor, and parkinsonism in a 54 year old man with 2-hydroxyglutaric aciduria. J Neurol Neurosurg Psychiatry 75:1362-1363. doi:10.1136/jnnp.2003.033571

27. Pascual-Castroviejo I, Pascual-Pascual SI, Velazquez Fragua R et al (2005) L-2 hydroxyglutaric aciduria in a patient with Klinefelter syndrome. Neurologia 20:90-93

28. Patay Z (2005) Diffusion-weighted MR imaging in leukodystrophies. Eur Radiol 15:2284-2303. doi:10.1007/s00330-005-2846-2

29. Periasamy V, Rudwan M, Yadav G et al (2008) Epilepsy in a young adult caused by L-2-hydroxyglutaric aciduria: a case report. Med Princ Pract 17:258-261. doi:10.1159/000117804

30. Rzem R, Van Schaftingen E, Veiga-da-Cunha M (2006) The gene mutated in L-2-hydroxyglutaric aciduria encodes L-2-hydroxyglutarate dehydrogenase. Biochimie 88(1):113-116. doi:10.1016/j. biochi.2005.06.005

31. Rzem R, Veiga-da-Cunha M, Noel G et al (2004) A gene encoding a putative FAD-dependent L-2-hydroxyglutarate dehydrogenase is mutated in L-2-hydroxyglutaric aciduria. Proc Natl Acad Sci U S A 101(48):16849-16854 . doi:10.1073/pnas.0404840101

32. Rzem R, Vincent MF, Van Schaftingen E et al (2007) L-2hydroxyglutaric aciduria, a defect of metabolite repair. J Inherit Metab Dis 30:681-689. doi:10.1007/s10545-007-0487-0

33. Samuraki M, Komai K, Hasegawa Y et al (2008) A successfully treated adult patient with L-2-hydroxyglutaric aciduria. Neurology 70:1051-1052. doi:10.1212/01.wnl.0000287141.90944.95

34. Sass JO, Jobard F, Topcu M et al (2008) L: -2-Hydroxyglutaric aciduria: identification of ten novel mutations in the L2HGDH gene. J Inherit Metab Dis. doi:10.1007/s10545-008-0855-4

35. Seijo-Martinez M, Navarro C, Castro del Rio M et al (2005) L-2hydroxyglutaric aciduria: clinical, neuroimaging, and neuropathological findings. Arch Neurol 62:666-670. doi:10.1001/archneur.62.4.666

36. Sener RN (2004) Diffusion magnetic resonance imaging patterns in metabolic and toxic brain disorders. Acta Radiol 45:561-570. doi:10.1080/02841850410006128

37. Sener RN (2003) L-2 hydroxyglutaric aciduria: proton magnetic resonance spectroscopy and diffusion magnetic resonance imaging findings. J Comput Assist Tomogr 27:38-43. doi:10.1097/ 00004728-200301000-00008 
38. Shafeghati Y, Vakili G, Entezari A (2006) L-2-hydroxyglutaric aciduria: a report of six cases and review of the literature. Arch Iran Med 9:165-169

39. Struys EA, Gibson KM, Jakobs C (2007) Novel insights into L-2hydroxyglutaric aciduria: mass isotopomer studies reveal 2-oxoglutaric acid as the metabolic precursor of L-2-hydroxyglutaric acid. J Inherit Metab Dis 30:690-693. doi:10.1007/s10545-0070697-5

40. Struys EA, Jansen EE, Verhoeven NM et al (2004) Measurement of urinary D- and L-2-hydroxyglutarate enantiomers by stableisotope-dilution liquid chromatography-tandem mass spectrometry after derivatization with diacetyl-L-tartaric anhydride. Clin Chem 50:1391-1395. doi:10.1373/clinchem.2004.033399

41. Sztriha L, Gururaj A, Vreken P et al (2002) L-2-hydroxyglutaric aciduria in two siblings. Pediatr Neurol 27:141-144. doi:10.1016/ S0887-8994(02)00405-8

42. Topcu M, Aydin OF, Yalcinkaya C et al (2005) L-2-hydroxyglutaric aciduria: a report of 29 patients. Turk J Pediatr 47:1-7
43. Vilarinho L, Cardoso ML, Gaspar P et al (2005) Novel L2HGDH mutations in 21 patients with L-2-hydroxyglutaric aciduria of Portuguese origin. Hum Mutat 26:395-396. doi:10.1002/humu.9373

44. Wanders RJ, Vilarinho L, Hartung HP et al (1997) L-2Hydroxyglutaric aciduria: normal L-2-hydroxyglutarate dehydrogenase activity in liver from two new patients. J Inherit Metab Dis 20:725-726. doi:10.1023/A:1005355316599

45. Wilcken B, Pitt J, Heath D et al (1993) L-2-hydroxyglutaric aciduria: three Australian cases. J Inherit Metab Dis 16:501-504. doi:10.1007/BF00711665

46. Yilmaz K (2008) Riboflavin treatment in a case with 1-2hydroxyglutaric aciduria. Eur J Paediatr Neurol (in press)

47. Zafeiriou DI, Sewell A, Savvopoulou-Augoustidou P et al (2001) L-2-Hydroxyglutaric aciduria presenting as status epilepticus. Brain Dev 23:255-257. doi:10.1016/S0387-7604(01)00206-6

48. Zafeiriou DI, Ververi A, Salomons GS et al (2008) L-2Hydroxyglutaric aciduria presenting with severe autistic features. Brain Dev 30:305-307. doi:10.1016/j.braindev.2007.09.005 